\title{
Cardiorespiratory Fitness is Inversely Associated with Risk of Low Bone Mineral Density in Older Korean Men
}

\author{
Inhwan Lee, Jeonghyeon Kim and Hyunsik Kang * (D) \\ College of Sport Science, Sungkyunkwan University, Suwon 16419, Korea; ansh00@naver.com (I.L.); \\ zzagkim115@naver.com (J.K.) \\ * Correspondence: hkang@skku.edu; Tel.: +82-31-299-6911
}

Received: 29 September 2020; Accepted: 26 October 2020; Published: 28 October 2020

check for updates

\begin{abstract}
Little is known regarding the association between physical fitness and bone health in older Korean men. This study investigated the relationship between estimated cardiorespiratory fitness (eCRF) and bone mineral density (BMD). This cross-sectional study included 2715 Korean men aged 50 years and older selected from those who participated in the 2008-2011 Korea National Health and Nutritional Examination and Survey. eCRF was obtained using a sex-specific algorithm developed on the basis of age, body mass index, resting heart rate, and physical activity and classified into low, middle, and high categories. Femoral neck BMD was assessed by dual X-ray absorptiometry. Odds ratios (OR) and 95\% confidence intervals (CI) for osteopenia, osteoporosis, and low BMD were calculated for eCRF categories in models fully adjusted for age, waist circumference, education, income, smoking, heavy alcohol intake, serum vitamin D, serum parathyroid hormone, and dietary intake of energy, protein, calcium, and vitamins A and C. Overall, eCRF levels were positively associated with BMD and negatively with prevalence of osteopenia, osteoporosis, and low BMD. Logistic regression showed inverse trends in the risks of osteopenia (high vs. low: OR $=0.692 ; 95 \%$ CI, 0.328-0.517; $p=0.049$ ) and low BMD (high vs. low: OR =0.669; 95\% CI, 0.497-0.966; $p=0.029$ ) by eCRF category in models fully adjusted for all the measured covariates. The current findings suggest that maintaining high eCRF via regular physical activity may contribute to attenuation of age-related loss of BMD and decreased risk for low BMD in older Korean men.
\end{abstract}

Keywords: physical activity; cardiorespiratory fitness; bone health; older adults

\section{Introduction}

Osteoporosis is a skeletal disease characterized by low bone mass and deterioration of bone structural integrity, leading to bone fragility and increasing the risk for bone fracture. Aging is an established risk factor of the disease, and its prevalence is predominantly in adults aged 50 years and older [1]. With a rapidly rising elderly population, osteoporosis has become a serious public health issue in both developed and developing countries [2]. The prevalence of osteoporosis is also associated with behavioral risk factors such as smoking, heavy alcohol consumption [3], dietary intake [4,5], vitamin D deficiency [6], and physical inactivity [7] in conjunction with low socio-economic status [8].

In South Korea, low bone mineral density (BMD) in older men is an underappreciated health issue. Older Korean men have a lower prevalence of osteoporosis and a lower standardized prevalence of vertebral fractures using the age distribution of the Korean population than older Korean women [9-11]. Interestingly enough, however, older Korean men are at higher risk of post-hip-fracture mortality compared with older Korean women [12,13], with the highest standardized mortality rates seen in the age group of 50-59 years [12]. By analyzing nationwide data involving Korean populations aged 50 
years and older, Ha [14] reported that the prevalence of osteoporosis ranged from $6.1 \%$ to $13.1 \%$ in men and from $24.3 \%$ to $35.5 \%$ in women. The incidence rate of osteoporotic fracture was lower in men than in women (i.e., 110.5/100,000 in men vs. 243.1/100,000 women. However, men had approximately 1.4and 2.2-fold higher risks of post-spinal-fracture mortality [15] (Kim et al., 2016) and post-hip-fracture mortality [16], respectively, compared with women. Given the relationship between osteoporosis and mortality, therefore, maintaining BMD is vital to men's bone health later in life.

Both resistance exercise and weight-bearing aerobic exercise are recommended as a primary therapeutic strategy for the prevention and treatment of low BMD, including osteopenia and osteoporosis [17]. Observational studies showed that physically active individuals had attenuated age-related decline in BMD compared with physically inactive ones [18]. In addition to physical activity, recent studies examined the relationship between cardiorespiratory fitness (CRF) and BMD in older adults and reported an attenuating effect of high CRF on age-related declines in BMD in older women [19] and men [20]. To the best of our knowledge, however, little is known regarding the role of CRF as a prognostic tool in determining the risk of osteopenia and osteoporosis in older Korean adults. Therefore, an assessment of the relationship between CRF and BMD will provide the opportunity to develop better therapeutic strategies for Korean patients with osteoporosis.

CRF reflects the maximal capacity of the respiratory and cardiovascular systems to supply oxygen to working skeletal muscles during exercise and represent an objective indicator of habitual physical activity. In older populations, however, there is limited evidence demonstrating the prognostic role of CRF in relation to bone health status. An objective assessment of CRF requires a direct measurement of oxygen consumption with gas analyzers and well-trained personnel during a graded exercise test, which is expensive, time-consuming, and often difficult to implement in a population-based study. Alternatively, age-sex-specific algorithms consisting of routinely obtained health measures have been developed to estimate CRF (eCRF) with acceptable accuracy [21]. Therefore, this study aimed to investigate the relationship between $\mathrm{eCRF}$ and BMD in a cohort of Korean men aged 50 years and older selected from the participants of the 2008-2011 Korea National Health and Nutritional Examination and Survey (KNHANES) IV and V.

\section{Materials and Methods}

\subsection{Data Source and Study Participants}

In this study, we focused only on men aged 50 years and older $(n=5872)$ from the 2008-2011 KNHANES IV and V, nationwide surveys designed to assess health and nutritional status of the Korean population. A detailed description of the KNHANES including the sampling design is available elsewhere [22].

We initially selected men aged 50 years and older $(n=4,010)$ who underwent dual-energy X-ray absorptiometry (DEXA)-based assessment of bone mineral density. We excluded women or men who had no data available regarding height and weight $(n=11)$, waist circumference $(n=12)$, income $(n=47)$, education $(n=8)$, physical activity $(n=36)$, or resting heart rate (RHR) $(n=421)$. Additionally, men with thyroid disease $(n=40)$, rheumatic arthritis $(n=47)$, mobility limitations (defined as any orthopedic or physical impairment and/or an inability to move) $(n=669)$, or chronic kidney diseases $(n=4)$ were also excluded. Consequently, a total of 2715 men were included in final data analyses (Figure 1). The Korea Centers for Disease Control and Prevention institutional review board reviewed and approved the 2008-2011 KNHANES IV and V surveys (2008-04EXP-01-C, 2009-01CON-03-2C, 2010-02CON-21-C, 2011-02CON-06-C) in accordance with the Declaration of Helsinki. Informed consent form was obtained from all the participants. 


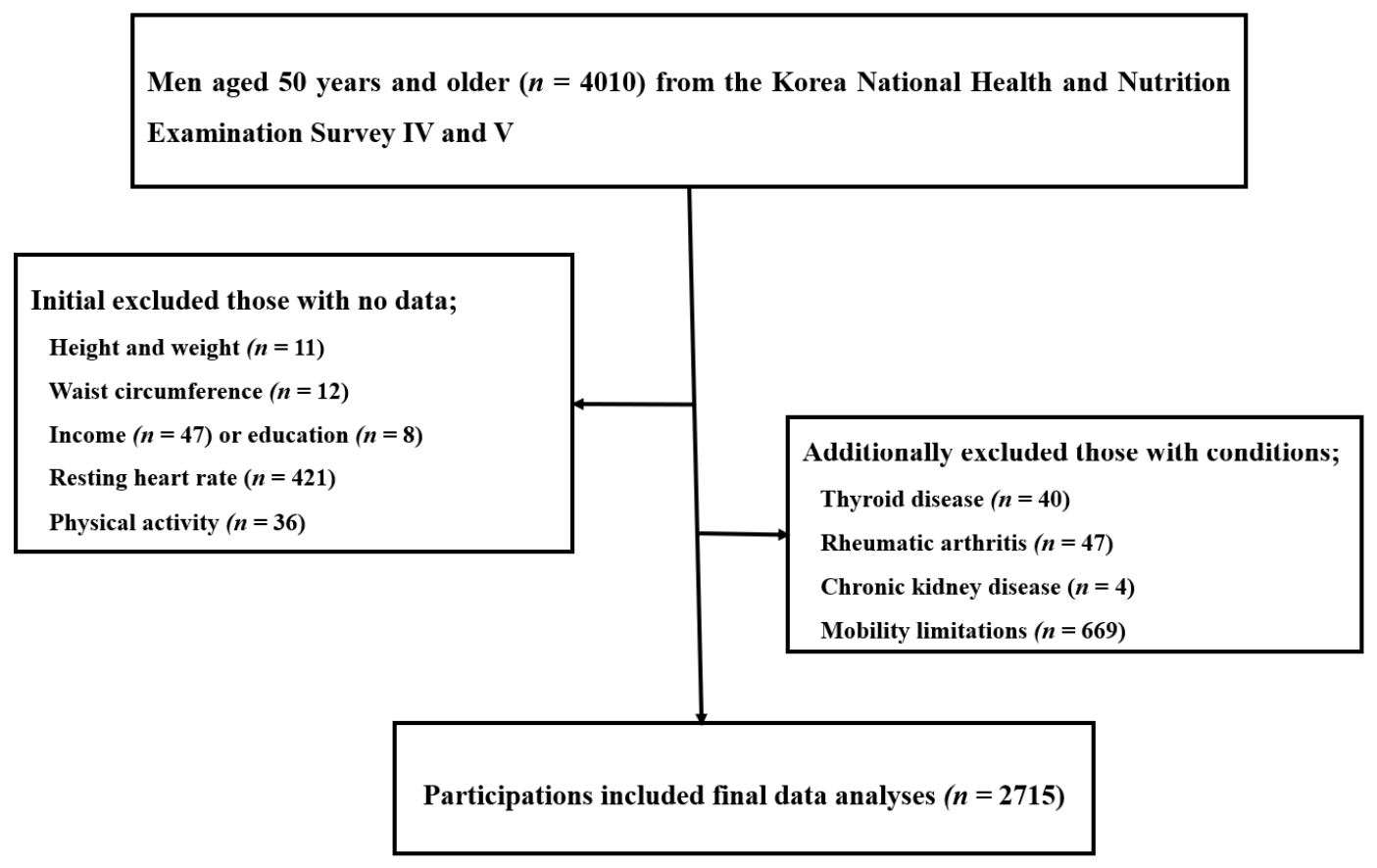

Figure 1. Selection of eligible study participants.

\subsection{Study Variables}

\subsubsection{Assessment of Bone Mineral Density}

BMD was measured at the femoral neck by DEXA with the Hologic Discovery QDR4500W bone density unit (Hologic Inc., Bedford, MA, USA). Quality control and calibration standards were conducted as specified by the manufacturer, with a precision coefficient of variation of $2.5 \%$. Osteoporosis was defined as a T-score of -2.5 or less, and osteopenia was defined as a T-score of -1.0 or less and greater than -2.5 . For regression modeling, T-score thresholds of -1.0 or less and greater than -2.5 (osteopenia), -2.5 or less (osteoporosis), and -1.0 or less (low BMD) were utilized. The reference values for BMD were determined from a reference group of young healthy adults aged 20-29 years in accordance with the recommendations of the World Health Organization (WHO) [9].

\subsubsection{Estimation of Cardiorespiratory Fitness}

Estimation of CRF (eCRF) was obtained in units of metabolic equivalents (METs) by using a sex-specific algorithm as previously reported [21]; eCRF (METs) $=2.77$ (sex) -0.10 (age) -0.17 (body mass index) -0.03 (resting heart rate) +1.00 (physical activity score) +18.07 . Physical activity score was assessed according to the Johnson Space Centre (JSC) Physical Activity Rating (PAR) scale [23] in the range of 0 (inactive) to 7 (highly active with more than $3 \mathrm{~h}$ per week). eCRF was classified into low (lowest 25\%), middle (middle 50\%), and high (highest 25\%).

\subsubsection{Determination of Covariates}

Height $(\mathrm{m})$ and weight $(\mathrm{kg})$ were measured using a digital mobile stadiometer (seca 274, SECA, Hamburg, Germany) and a portable bench scale (GL-6000-20, G-tech, Seoul, Republic of Korea), respectively, with light clothing and no shoes. Body mass index (BMI) was calculated in unit of weight $(\mathrm{kg}) /$ height $\left(\mathrm{m}^{2}\right)$. Waist circumference (WC in unit of $\mathrm{cm}$ ) was measured at the narrowest location between the iliac crest and the lower border of the rib cage using a retractable measuring tape (seca 201, SECA, Hamburg, Germany). Parameters of socio-economic status and health behaviors, such as education (i.e., elementary, middle, and high school, and college and higher), household income, smoking (past and current smokers), and heavy drinking (seven or more standard drinks with a 
frequency of two times or more per month), were assessed with the health interview questionnaire described previously [22]. Blood samples were collected from antecubital veins after an overnight fast to assess serum levels of 25-hydroxyvitamin D3 (25(OH)D) and parathyroid hormone (PTH) by radioimmunoassay (1470 Wizard gamma counter; PerkinElmer, Turku, Finland) and the N-tact PTH assay with LIAISON (Diasorin ${ }^{\circledR}$ Analyzer, Centralino, Italy), respectively. A face-to-face interview by a well-trained nutritionist was performed to assess dietary intakes of daily total energy, protein, calcium, and vitamin $\mathrm{A}$ and $\mathrm{C}$ supplements. A detailed description of the health and nutritional examinations and blood chemistry profiles is available elsewhere [22].

\subsection{Statistical Analyses}

All statistics of this survey have been calculated using sample weights assigned to sample participants [22]. Data are presented as mean \pm standard deviation (SD) and percentage (\%) for continuous and categorical variables, respectively. The Cochran-Mantel-Haenszel test and linear regression were used to test any significant trends in categorical and continuous variables, respectively, according to eCRF category (from low to high). Logistic regression was used to calculate odds ratio (ORs) and 95\% confidence intervals (CIs) for osteopenia, osteoporosis, and low BMD according to eCRF category (from low to high). Statistical significance was tested at $p=0.05$. All statistical analyses were conducted with the SPSS-PC statistical software (version 23.0, SPSS, IBM, Armonk, NY, USA).

\section{Results}

Table 1 represents the characteristics of the study participants by eCRF category. Negative linear trends were found in age $(p<0.001)$, BMI $(p<0.001)$, WC $(p<0.001)$, RHR $(p<0.001)$, and heavy alcohol consumption $(p<0.001)$ by eCRF category (from low to high). The middle and high eCRF groups were younger and had lower means of BMI, WC, RHR, and heavy alcohol intake compared with the low eCRF group. On the other hand, the middle and high eCRF groups were more active $(p<0.001)$ and had higher means of smoking $(p<0.001)$ and education $(p<0.001)$ compared with the low eCRF group.

With respect to blood chemistry and nutrients, positive linear trends were observed in vitamin $\mathrm{D}$ $(p=0.004)$, energy intake $(p<0.001)$, protein $(p<0.001)$, calcium $(p<0.001)$, vitamin A $(p<0.001)$, and vitamin $C(p<0.001)$ by eCRF category. The middle and high eCRF category groups had higher values of serum vitamin $\mathrm{D}$, caloric intake, and daily intakes of protein, calcium, vitamin A, and vitamin D, compared with the low eCRF category group. On the other hand, an inverse linear trend was observed in serum PTH $(p<0.001)$ by eCRF category. The middle and high eCRF category groups had a lower level of serum PHT compared with the low eCRF category group.

Table 2 describes BMDs and T-scores of the femoral neck by eCRF category. Positive linear trends were found in BMD $(p<0.001)$ and T-score $(p<0.001)$ by eCRF category (from low to high). The middle and high eCRF category groups had higher BMD and T-scores compared with the low eCRF category group. In addition, inverse linear trends were observed in the prevalence of osteopenia $(p<0.001)$ and osteoporosis $(p<0.001)$ by eCRF category. The middle and high eCRF category groups had a lower prevalence of osteopenia and osteoporosis compared with the low eCRF category group. 
Table 1. Description of measured parameters according to estimated cardiorespiratory fitness (eCRF) levels.

\begin{tabular}{|c|c|c|c|c|c|}
\hline \multirow{2}{*}{ Variables } & \multirow{2}{*}{$\begin{array}{c}\text { All } \\
(n=2715)\end{array}$} & \multicolumn{3}{|c|}{ eCRF Levels } & \multirow{2}{*}{$p$ for Linear Trends } \\
\hline & & $\begin{array}{c}\text { Low } \\
(n=678)\end{array}$ & $\begin{array}{l}\text { Middle } \\
(n=1351)\end{array}$ & $\begin{array}{c}\text { High } \\
(n=686)\end{array}$ & \\
\hline \multicolumn{6}{|l|}{ Anthropometrics } \\
\hline Age (years) & $62.5 \pm 8.6$ & $70.6 \pm 7.6$ & $61.5 \pm 7.6$ & $56.4 \pm 5.2$ & $<0.001$ \\
\hline Body mass index $\left(\mathrm{kg} / \mathrm{m}^{2}\right)$ & $23.8 \pm 2.9$ & $24.2 \pm 3.1$ & $23.8 \pm 3.0$ & $23.3 \pm 2.5$ & $<0.001$ \\
\hline Waist circumference (cm) & $85.2 \pm 8.5$ & $87.5 \pm 9.3$ & $85.3 \pm 8.5$ & $82.9 \pm 7.2$ & $<0.001$ \\
\hline Resting heart rate (beats/min) & $70 \pm 9$ & $72 \pm 10$ & $70 \pm 9$ & $67 \pm 8$ & $<0.001$ \\
\hline Heavy alcohol drinkers, $n(\%)$ & $609(22.4)$ & $232(38.1)$ & $265(43.5)$ & $112(18.4)$ & $<0.001$ \\
\hline Current smokers, $n(\%)$ & $1589(70.2)$ & $366(23.0)$ & $779(40.9)$ & $444(27.9)$ & $<0.001$ \\
\hline \multicolumn{6}{|l|}{ Socio-economic Status } \\
\hline Education, $n(\%)$ & & & & & $<0.001$ \\
\hline Elementary & $873(32.3)$ & $317(36.3)$ & $407(46.5)$ & $150(17.2)$ & \\
\hline Middle and high schools & $1323(48.7)$ & $271(20.5)$ & $682(51.5)$ & $370(28.0)$ & \\
\hline College or higher & $519(19.1)$ & $90(17.3)$ & $263(50.7)$ & $166(32.0)$ & \\
\hline Household income (won) & $340 \pm 1172$ & $210 \pm 405$ & $340 \pm 778$ & $469 \pm 2015$ & $<0.001$ \\
\hline \multicolumn{6}{|l|}{ Physical Activity and Fitness } \\
\hline Physical activity score & $3.36 \pm 1.55$ & $1.83 \pm 0.54$ & $3.31 \pm 1.48$ & $4.95 \pm 0.21$ & $<0.001$ \\
\hline eCRF (METs) & $10.0 \pm 1.7$ & $7.9 \pm 0.6$ & $10.0 \pm 0.9$ & $12.2 \pm 0.5$ & $<0.001$ \\
\hline \multicolumn{6}{|l|}{ Serum Vitamin and Hormones } \\
\hline Vitamin D $(\mathrm{ng} / \mathrm{mL})$ & $21.5 \pm 7.5$ & $21.1 \pm 7.7$ & $21.3 \pm 7.3$ & $22.1 \pm 7.6$ & 0.004 \\
\hline Parathyroid hormone $(p \mathrm{~g} / \mathrm{mL})$ & $65.5 \pm 26.4$ & $69.1 \pm 31.7$ & $65.0 \pm 24.6$ & $63.5 \pm 24.1$ & $<0.001$ \\
\hline \multicolumn{6}{|l|}{ Nutrient intake } \\
\hline Energy (kcal/day) & $2139 \pm 789$ & $1904 \pm 655$ & $2166 \pm 803$ & $2340 \pm 828$ & $<0.001$ \\
\hline Protein (g/day) & $75 \pm 35$ & $64 \pm 30$ & $76 \pm 36$ & $83 \pm 36$ & $<0.001$ \\
\hline Calcium (mg/day) & $554 \pm 330$ & $488 \pm 361$ & $575 \pm 399$ & $585 \pm 330$ & $<0.001$ \\
\hline Vitamin A ( $\mu \mathrm{gRE})$ & $847 \pm 876$ & $706 \pm 770$ & $864 \pm 811$ & $966 \pm 1,074$ & $<0.001$ \\
\hline Vitamin C (mg/day) & $111 \pm 92$ & $94 \pm 79$ & $116 \pm 99$ & $120 \pm 88$ & $<0.001$ \\
\hline
\end{tabular}

eCRF: estimated cardiorespiratory fitness; METs: metabolic equivalents. $p$ values of less than 0.05 indicate significant linear trends according to incremental eCRF categories (from low to high). 
Table 2. Femoral neck bone mineral density (BMD) by eCRF category.

\begin{tabular}{|c|c|c|c|c|c|}
\hline \multirow{2}{*}{ Variables } & \multirow{2}{*}{$\begin{array}{c}\text { All } \\
(n=2715)\end{array}$} & \multicolumn{3}{|c|}{ eCRF Levels } & \multirow{2}{*}{$p$ for Trends } \\
\hline & & $\begin{array}{c}\text { Low } \\
(n=678)\end{array}$ & $\begin{array}{c}\text { Middle } \\
(n=1351)\end{array}$ & $\begin{array}{c}\text { High } \\
(n=686)\end{array}$ & \\
\hline $\operatorname{BMD}\left(\mathrm{g} / \mathrm{cm}^{2}\right)$ & $0.75 \pm 0.11$ & $0.71 \pm 0.12$ & $0.75 \pm 0.12$ & $0.78 \pm 0.11$ & $<0.001$ \\
\hline T-score $($ mean $\pm \mathrm{SD})$ & $-0.76 \pm 0.95$ & $-1.06 \pm 0.98$ & $-0.74 \pm 0.98$ & $-0.51 \pm 0.88$ & $<0.001$ \\
\hline Osteopenia $(n,[\%])$ & $1044(38.5)$ & $320(47.2)$ & $524(38.8)$ & $200(29.2)$ & $<0.001$ \\
\hline Osteoporosis $(n,[\%])$ & $71(2.6)$ & $41(6.0)$ & $25(1.9)$ & $5(0.7)$ & $<0.001$ \\
\hline Low $\operatorname{BMD}(n,[\%])$ & 1115 (41.1) & $361(53.2)$ & $549(40.6)$ & $205(29.9)$ & $<0.001$ \\
\hline $\operatorname{Normal}(n,[\%])$ & $1600(60.5)$ & $317(49.8)$ & $802(60.5)$ & $481(70.6)$ & $<0.001$ \\
\hline
\end{tabular}

eCRF: estimated cardiorespiratory fitness; osteopenia is defined as a T-score of -1.0 or less and greater than -2.5, and osteoporosis is defined as a T-score of -2.5 or less; low BMD includes both osteopenia and osteoporosis; the cut-off eCRF values were $\leq 7.9$ METS for low, 8.0 12.1 METs for middle, and $\geq 12.2$ METs for high groups.

Table 3 represents the odds ratios for osteopenia, osteoporosis, and low BMD by eCRF category (from low to high). The middle and high eCRF groups had ORs of $0.647(p<0.001)$ and 0.412 $(p<0.001)$ for osteopenia, respectively compared with the low eCRF group $(\mathrm{OR}=1)$. The OR for the high category group remained statistically significant $(p=0.049)$ even after adjustments for all the measured covariates. In addition, the middle and high eCRF groups had ORs of $0.241(p<0.001)$ and $0.080(p<0.001)$ for osteoporosis, respectively compared with the low eCRF group. The ORs for the middle and high eCRF groups were no longer significant after adjustments for all the measured covariates. Finally, the middle and high eCRF groups had $40 \%(p<0.001)$ and $63 \%(p<0.001)$ lower risks, respectively, of low BMD compared with the low eCRF group. The OR for the high eCRF group remained significant $(p=0.029)$ even after adjustments for all the measured covariates.

Table 3. Odds ratios (OR) and 95\% confidence intervals (CI) for osteopenia, osteoporosis, and low bone mineral density by eCRF category.

\begin{tabular}{|c|c|c|c|c|c|}
\hline Variables & Low eCRF & Middle eCRF & $p$ Value & High eCRF & $p$ Value \\
\hline \multicolumn{6}{|l|}{ Osteopenia } \\
\hline Crude OR $(95 \%$ CI $)$ & 1 & $\begin{array}{c}0.647 \\
(0.535-0.783)\end{array}$ & $<0.001$ & $\begin{array}{c}0.412 \\
(0.328-0.517)\end{array}$ & $<0.001$ \\
\hline Adjusted a OR (95\% CI) & 1 & $\begin{array}{c}0.923 \\
(0.703-1.213)\end{array}$ & 0.565 & $\begin{array}{c}0.692 \\
(0.480-0.998)\end{array}$ & 0.049 \\
\hline \multicolumn{6}{|l|}{ Osteoporosis } \\
\hline Crude OR $(95 \% \mathrm{CI})$ & 1 & $\begin{array}{c}0.241 \\
(0.144-0.403)\end{array}$ & 0.001 & $\begin{array}{c}0.080 \\
(0.031-0.206)\end{array}$ & 0.001 \\
\hline Adjusted a OR $(95 \%$ CI $)$ & 1 & $\begin{array}{c}0.708 \\
(0.308-1.632)\end{array}$ & 0.418 & $\begin{array}{c}0.354 \\
(0.089-1.413)\end{array}$ & 0.142 \\
\hline \multicolumn{6}{|l|}{ Low Bone Mineral Density } \\
\hline Crude OR $(95 \% \mathrm{CI})$ & 1 & $\begin{array}{c}0.601 \\
(0.499-0.724)\end{array}$ & $<0.001$ & $\begin{array}{c}0.374 \\
(0.300-0.407)\end{array}$ & $<0.001$ \\
\hline Adjusted a OR $(95 \%$ CI $)$ & 1 & $\begin{array}{c}0.905 \\
(0.691-1.184)\end{array}$ & 0.467 & $\begin{array}{c}0.669 \\
(0.497-0.966)\end{array}$ & 0.029 \\
\hline
\end{tabular}

eCRF, estimated cardiorespiratory fitness; BMD, bone mineral density. Osteopenia is defined as a T-score of -1.0 or less and greater than -2.5 , and osteoporosis is defined as a T-score of -2.5 or less; low BMD includes both osteopenia and osteoporosis. ${ }^{a}$ Model adjusted for age, education, household income, waist circumference, heavy alcohol intake, smoking, serum vitamin $\mathrm{D}$, serum parathyroid hormone, caloric intake, protein intake, calcium intake, vitamin A intake, and vitamin C intake.

\section{Discussion}

In this study, we examined the relationship between eCRF and femoral neck BMD in a sample of 2715 Korean men extracted from those who participated in the 2008-2011 KHHANES IV and V. We are the first to report that eCRF is significantly and inversely associated with risks of osteopenia and osteoporosis in otherwise healthy older Korean men. In addition, the findings of this study showed 
that low BMD at the femoral neck is a widespread problem in older Korean men with a prevalence of approximately $41 \%$.

The findings of the study agreed with previous studies that reported the association between higher CRF and better bone health in older adults. For example, Wainstein et al. [20] examined the association between objectively measured CRF and BMD at the femoral neck in 2569 men aged 50-90 years and found that higher BMD was significantly associated with lower risks for osteoporosis and low BMD. Schwarz et al. [24] examined the relationships of muscular strength and CRF with BMD at the total hip and lumbar spine in 153 Danish men aged 31 to 60 years. Objectively measured CRF was positively associated with the T-scores of the total hip and lumbar spine in models fully adjusted for age, weight, height, smoking, alcohol intake, and leisure time physical activity. The positive association between CRF and BMD was also reported from a cohort of community-dwelling 802 elderly Portuguese men and women [25]. Together, these findings suggest that having or maintaining high CRF is significantly associated with attenuated age-related loss in BMD and a lower risk of osteopenia and/or osteoporosis in older men.

CRF indicative of habitual physical activity has been used as an alternative to examine the association between physical activity and bone health. However, a majority of previous studies examining the relationship between physical activity and bone health have been conducted in women and showed that both resistance and weight-bearing aerobic exercises are significantly associated with increased bone mass and strength [26], attenuated aged-related loss in BMD [16], and decreased risk for bone fracture [27]. Unlike women, however, evidence is not enough to recommend resistance or weight-bearing exercise as a nonpharmacologic strategy for improving or maintaining BMD in older men $[28,29]$. For example, previous studies examining the effects of exercise training on BMD in men led to conflicting results due to small sample sizes [30]. Other studies were conducted in healthier and younger men with better bones and fewer covariates [31,32]. In this aspect, we believe that the current study is an important addition to the literature, by reporting the relationship between eCRF and bone health in a large sample of older men in models fully adjusted for covariates.

Several explanations can be given for the current study findings. First, the attenuating effect of high eCRF against the risk of low BMD may be attributed to physical activity-induced increase of mechanical loading and stimulation of new bone formation at the stressed skeletal sites. Participation of regular physical activity results in a number of bone health benefits, including increases in BMD and bone size and increases cortical area and strength during adolescence [33] and adulthood [34], as well as reduced risk for bone fracture later in life [35].

Second, higher serum vitamin D in conjunction with lower serum PTH level by eCRF category (from low to high) may explain the decreased risk of low BMD observed among individuals with middle and/or high eCRF levels. Serum vitamin D level is inversely correlated with serum PTH level; Vitamin D insufficiency causes an increase in serum PTH and in mineral release from bone. In the meantime, chronically elevated serum PTH secondary to vitamin D insufficiency may stimulate bone turnover, causing negative bone balance and increased risks for low BMD and bone fracture. We speculate that higher serum vitamin D levels observed in the middle and high eCRF groups compared with the low eCRF group may be due to group differences in body fat [36] or nutritional intake [37] or outdoor activity [38,39]. In particular, the importance of the nutritional intake of vitamins D and B and calcium in relation to bone health was previously reported from recent studies using a nationwide survey data [6,37].

Lastly, the relationship between eCRF and femoral neck BMD may be influenced by several covariates such as genetics, growth factors, gender, age, body composition, health behaviors (i.e., smoking and alcohol intake), medications, hormones, and nutrition [40]. In this study, the association between higher eCRF and lower risk of low BMD was tested in a model fully adjusted for age, SES, WC, smoking, heavy alcohol intake, serum hormones (vitamin D and PTH), and dietary factors (caloric intake, protein, calcium, and vitamins $\mathrm{A}$ and $\mathrm{C}$ ), minimizing the influence of the covariates on the relationship between eCRF and risk of low BMD. 
This study has limitations. First, any causal inference on the relationship between eCRF and BMD cannot be possible. Second, a bidirectional relationship between CRF and BMD can be possible. That is, younger men and/or men with better overall health are more likely to participate in regular physical activity and be physically fit and have healthier bone. Third, the cellular and molecular mechanisms by which high CRF decreases the risk for low BMD remain to be elucidated. Lastly, the validity of the eCRF algorithm used in this study should be confirmed in older Korean populations.

Despite the limitations, we believe that the current findings of the study support and extend the literature regarding physical fitness and bone health by reporting the relationship between higher eCRF and lower risk for osteopenia and osteoporosis in a higher risk group of advanced age in models fully adjusted for a number of potential covariates.

\section{Conclusions}

In this cross-sectional study, we examined the relationship between eCRF and BMD in 2715 older Korean adults aged 50 years and older and showed that higher eCRF was significantly associated with attenuated age-related loss in BMD and decreased risk of low BMD. Together, the current findings of the study have some practical and clinical implications. First, maintaining high CRF via regular exercise is important for the maintenance of good bone health and the prevention of osteoporosis and fracture as well as premature death from hip fracture in older Korean men. Second, assessment of eCRF using routinely obtained health measures is highly feasible in clinics and hospitals and should be considered as a diagnostic tool for bone health status.

Author Contributions: I.L. and H.K. contributed to the conception and design, data analyses, data interpretation, and writing of the manuscript. J.K. contributed to data analyses and writing of the manuscript (first draft). All authors have read and agreed to the published version of the manuscript.

Funding: This work was supported by the National Research Foundation Grant funded by the Korean Government (NRF-2019R1I1A1A01043771).

Acknowledgments: We gratefully thank the Korea Centers for Disease Control and Prevention (KCDC) for allowing use of their data for this study.

Conflicts of Interest: The authors declare no conflict of interest to disclose.

\section{References}

1. Lee, E.Y.; Kim, D.; Kim, K.M.; Kim, K.J.; Choi, H.S.; Rhee, Y.; Lim, S.-K. Age-related bone mineral density patterns in Koreans (KNHANES IV). J. Clin. Endocrinol. Metab. 2012, 97, 3310-3318. [PubMed]

2. Kim, J.-W.; Ha, Y.-C.; Lee, Y.-K. Factors affecting bone mineral density measurement after fracture in South Korea. J. Bone Metab. 2017, 24, 217-222. [CrossRef] [PubMed]

3. Jang, H.-D.; Hong, J.-Y.; Han, K.; Lee, J.C.; Shink, B.-J.; Choi, S.-W.; Suh, S.-W.; Yang, J.-H.; Park, S.-Y.; Bang, C. Relationship between bone mineral density and alcohol intake: A nationwide health survey analysis of postmenopausal women. PLoS ONE 2017, 12, e0180132. [CrossRef]

4. Ha, J.; Kim, A.-A.; Lim, K.; Shin, S. The association of potassium intake with bone mineral density and the prevalence of osteoporosis among older Korean adults. Nutr. Res. Pract. 2020, 14, 55-61. [CrossRef] [PubMed]

5. Choi, E.; Park, Y. The association between the consumption of fish/shellfish and the risk of osteoporosis in men and postmenopausal women aged 50 years or older. Nutrients 2016, 8, 113. [CrossRef] [PubMed]

6. Yoo, K.-O.; Kim, M.-J.; Ly, S.-Y. Association between vitamin D intake and bone mineral density in Koreans aged $\geq 50$ years: Analysis of the 2009 Korea National Health and Nutrition Examination Survey using a newly established vitamin D database. Nutr. Res. Pract. 2019, 13, 115-125. [CrossRef]

7. Chang, H.K.; Chang, D.-G.; Myong, J.-P.; Kim, J.-H.; Lee, S.-J.; Lee, Y.S.; Lee, H.-N.; Lee, K.H.; Park, D.C.; Kim, C.J.; et al. Bone mineral density among Korean females aged 20-50 years: Influence of age at menarche (The Korea National Health and Nutrition Examination Survey 2008-2011). Osteoporos. Int. 2017, 28, 2129-2136. [CrossRef] 
8. Lee, S.H.; Kwon, H.-Y. Prevalence of Osteoporosis in Korean Patients with Chronic Obstructive Pulmonary Disease and Their Health-related Quality of Life According to the Korea National Health and Nutrition Examination Survey 2008-2011. J. Bone Metab. 2017, 24, 241-248. [CrossRef]

9. Lee, K.-S.; Bae, S.-H.; Lee, S.H.; Lee, J.; Lee, D.R. New Reference Data on Bone Mineral Density and the Prevalence of Osteoporosis in Korean Adults Aged 50 Years or Older: The Korea National Health and Nutrition Examination Survey 2008-2010. J. Korean Med. Sci. 2014, 29, 1514-1522. [CrossRef]

10. Park, E.J.; Joo, I.W.; Jang, M.-J.; Kim, Y.T.; Oh, K.; Oh, H.J. Prevalence of osteoporosis in the Korean population based on Korea National Health and Nutrition Examination Survey (KNHANES), 2008-2011. Yonsei Med. J. 2014, 55, 1049-1057. [CrossRef]

11. Ha, Y.-C.; Park, Y.-G.; Nam, K.-W.; Kim, S.-R. Trend in hip fracture incidence and mortality in Korea: A prospective cohort study from 2002 to 2011. J. Korean Med. Sci. 2015, 30, 483-488. [CrossRef] [PubMed]

12. Yoon, H.-K.; Park, C.; Jang, S.; Jang, S.; Lee, Y.-K.; Ha, Y.-C. Incidence and mortality following hip fracture in Korea. J. Korean Med Sci. 2011, 26, 1087-1092. [CrossRef] [PubMed]

13. Hong, S.; Han, K. The incidence of hip fracture and mortality rate after hip fracture in Korea: A nationwide population-based cohort study. Osteoporos. Sarcopenia 2019, 5, 38-43. [CrossRef] [PubMed]

14. Ha, Y.-C. Epidemiology of osteoporosis in Korea. J. Korean Med. Assoc. 2016, 59, 836-841. [CrossRef]

15. Kim, T.Y.; Jang, S.; Park, C.M.; Lee, A.; Lee, Y.K.; Kim, H.Y.; Cho, E.H.; Ha, Y.C. Trends of incidence, mortality, and future projection of spinal fractures in Korea using nationwide claims data. J. Korean Med. Sci. 2016, 31, 801-805. [CrossRef]

16. Ha, Y.C.; Kim, T.Y.; Lee, A.; Lee, Y.K.; Kim, H.Y.; Kim, J.H.; Park, C.M.; Jang, S. Current trends and future projections of hip fracture in South Korea using nationwide claims data. Osteoporos. Int. 2016, 27, 2603-2609. [CrossRef]

17. Kohrt, W.M.; Bloomfield, S.A.; Little, K.D.; Nelson, M.E.; Yingling, V.R. American College of Sports Medicine Position Stand: Physical activity and bone health. Med. Sci. Sports Exerc. 2004, 36, 1985-1996. [CrossRef]

18. Moayyeri, A. The association between physical activity and osteoporotic fractures: A review of the evidence and implications for future research. Ann. Epidemiol. 2008, 18, 827-835. [CrossRef]

19. DeFina, L.F.; Leonard, D.; Willis, B.L.; Barlow, C.E.; Finley, C.E.; Jenkins, M.R.; Pence, B.C.; Zhang, Y.; Chyu, M.-C.; Lewiecki, E.M.; et al. High cardiorespiratory fitness is associated with reduced risk of low bone density in postmenopausal women. J. Women's Health 2016, 25, 1073-1080. [CrossRef]

20. Wainstein, H.M.; Feldman, M.; Shen, C.L.; Leonard, D.; Willis, B.L.; Finley, C.E.; Gruntmanis, U.; DeFina, L.F. The Relationship Between Cardiorespiratory Fitness and Bone Mineral Density in Men: A Cross-sectional Study. Mayo Clin. Proc. 2016, 91, 726-734. [CrossRef]

21. Lee, I.; Kim, J.; Kang, H. Estimated cardiorespiratory fitness attenuates the impacts of sarcopenia and obesity on non-alcoholic fatty liver in Korean adults. Int. J. Environ. Res. Public Health 2020, 17, 3902. [CrossRef] [PubMed]

22. Kweon, S.; Kim, Y.; Jang, M.-J.; Kim, Y.; Kim, K.; Choi, S.; Chun, C.; Khang, Y.-H.; Oh, K. Data resource profile: The Korea National Health and Nutrition Examination Survey (KNHANES). Int. J. Epidemiol. 2014, 43, 69-77. [CrossRef] [PubMed]

23. Ross, R.M.; Jackson, A.S. Exercise Concepts, Calculations, and Computer Applications; Benchmark Press: Carmel, IN, USA, 1990; pp. 95-109.

24. Schwarz, P.; Jørgensen, N.; Nielsen, B.; Laursen, A.S.; Linneberg, A.; Aadahl, M. Muscle strength, power and cardiorespiratory fitness are associated with bone mineral density in men aged 31-60 years. Scan. J. Public Health 2014, 42, 773-779. [CrossRef] [PubMed]

25. Gouveia, É.R.; Maia, J.A.; Beunen, G.P.; Blimkie, C.J.; Rodrigues, A.L.; Freitas, D.L. Functional fitness and bone mineral density in the elderly. Arch. Osteoporos. 2012, 7, 75-85. [CrossRef]

26. Kelley, G.A. Exercise and regional bone mineral density in postmenopausal women: A meta-analytic review of randomized trials. Am. J. Phys. Med. Rehabil. 1998, 77, 76-87. [CrossRef] [PubMed]

27. Feskanich, D.; Willett, W.; Colditz, G. Walking and leisure time activity and risk of hip fracture in postmenopausal women. JAMA 2002, 288, 2300-2306. [CrossRef]

28. Kelley, G.A.; Kelley, K.S.; Tran, Z.V. Exercise and bone mineral density in men: A meta-analysis. J. Appl. Physiol. 2000, 88, 1730-1736. [CrossRef]

29. Kelley, G.A.; Kelley, K.S.; Kohrt, W.M. Exercise and bone mineral density in men: A meta-analysis of randomized controlled trials. Bone 2013, 53, 103-111. [CrossRef] 
30. Stewart, K.J.; Deregis, J.R.; Turner, K.L.; Bacher, A.C.; Sung, J.; Hees, P.S.; Tayback, M.; Ouyang, P. Fitness, fatness and activity as predictors of bone mineral density in older persons. J. Intern. Med. 2002, 252, 381-388. [CrossRef]

31. Huuskonen, J.; Vaisanen, S.B.; Kroger, H.; Jurvelin, C.; Bouchard, C.; Alhava, E.; Rauramaa, R. Determinants of bone mineral density in middle aged men: A population-based study. Osteoporos Int. 2000, 11, 702-708. [CrossRef]

32. Huuskonen, J.; Vaisanen, S.B.; Kroger, H.; Jurvelin, J.S.; Alhava, E.; Rauramaa, R. Regular physical exercise and bone mineral density: A four-year controlled randomized trial in middle-aged men: The DNASCO study. Osteoporos. Int. 2001, 12, 349-355. [PubMed]

33. Baxter-Jones, A.D.; Kontulainen, S.A.; Faulkner, R.A.; Bailey, D.A. A longitudinal study of the relationship of physical activity to bone mineral accrual from adolescence to young adulthood. Bone 2008, 43, 1101-1107.

34. Nilsson, M.; Ohlsson, C.; Mellström, D.; Lorentzon, M. Previous sport activity during childhood and adolescence is associated with increased cortical bone size in young adult men. J. Bone Miner. Res. 2009, 24, 125-133. [PubMed]

35. Michaelsson, K.; Olofsson, H.; Jensevik, K.; Larsson, S.; Mallmin, H.; Berglund, L.; Vessby, B.; Melhus, H. Leisure physical activity and the risk of fracture in men. PLoS Med. 2007, 4, e199. [CrossRef]

36. Han, S.S.; Kim, M.; Lee, S.M.; Lee, J.P.; Kim, S.; Joo, K.W.; Lim, C.S.; Kim, Y.S.; Kim, D.K. Association between body fat and vitamin D status in Korean adults. Asia Pac. J. Clin. Nutr. 2014, 23, 65-75.

37. Kim, H.; Kim, H.; Kwon, O.; Park, H. Food and nutrient intake level by the risk of osteoporosis and cardiovascular disease in postmenopausal women: The use of the 5th Korean National Health and Nutrition Examination Surveys (2010-2011). Korean J. Community Nutr. 2019, 24, 152-162.

38. Yoon, J.-S.; Song, M.-K. Vitamin D intake, Outdoor Activity Time and Serum 25-OH Vitamin D Concentrations of Korean Postmenopausal Women by Season and by Age. Korean J. Community Nutr. 2015, 20, 120-128.

39. Joh, H.-K.; Lim, C.S.; Cho, B. Lifestyle and dietary factors associated with serum 25-hydroxyvitamin D levels in Korean young adults. J. Korean Med Sci. 2015, 30, 1110-1120.

40. Khan, K.; McKay, H.; Kannus, P.; Bailey, D.; Wark, J.; Bennell, K. Physical Activity and Bone Health; Human Kinetics: Champaign, IL, USA, 2001.

Publisher's Note: MDPI stays neutral with regard to jurisdictional claims in published maps and institutional affiliations.

(C) 2020 by the authors. Licensee MDPI, Basel, Switzerland. This article is an open access article distributed under the terms and conditions of the Creative Commons Attribution (CC BY) license (http://creativecommons.org/licenses/by/4.0/). 